\title{
Effet du greffage horticole et de l'inoculation mycorhizienne sur la croissance du baobab (Adansonia digitata L.) en Moyenne et Haute Casamance (Sénégal)
}

Tamsir Mbaye,
(Chargé de Recherches, PhD, Phytogéographie)
Institut Sénégalais de Recherches Agricoles/Sénégal

Fatou Gning,

(Doctorant en Geoscience and Natural Resource Management)

Université de Copenhague

Dioumacor Fall,

(Chargé de Recherches, $\mathrm{PhD}$, Microbiologie/physiologie des plantes)

Institut Sénégalais de Recherches Agricoles/Sénégal

Ababacar Ndiaye,

(Doctorant en Agroforesterie et Productions Végétales)

Université Assane Seck de Ziguinchor/Sénégal

Daouda Ngom,

(Professeur, PhD, écologie)

Université Cheikh Anta DIOP de Dakar/Sénégal

Mahani Cisse,

(Master, Agroforesterie),

World vision Sénégal

Saliou Ndiaye,

(Professeur, $\mathrm{PhD}$, Agronomie)

Université Thiès/ENSA/Sénégal

Doi:10.19044/esj.2019.v15n36p507 URL:http://dx.doi.org/10.19044/esj.2019.v15n36p507

\section{Résumé}

L'objectif de ce travail est d'étudier l'effet du greffage et de l'inoculation mycorhizienne sur la croissance du baobab dans les régions de Kolda et de Sédhiou. Pour ce faire, un dispositif experimental en bloc aléatoire complet constitué de quatre traitements sur des plants de baobab (greffé inoculé, greffé non inoculé, non greffé inoculé et non greffé non inoculé) a été mis en place dans huit (8) sites. Le greffage a été effectué avec la méthode de fente terminale et l'inoculation avec un mélange de souches de champignons mycorhriziens arbusculaires (Glomus aggretatum, $G$. 
intraradices et G. Fasciculatum) en pépinière. Deux ans après plantation, les traitements ont montré que les plus gros diamètres et le plus grand nombre de rameaux sont obtenus avec les plants greffés (inoculés et non inoculés) alors que les hauteurs les plus élevées avec ceux non inoculés (non greffés et greffés). Le nombre de feuilles le plus imporatant est observé sur les plants non greffés non inoculés. Par ailleurs, des différences significatives entre les sites ont ausssi été constatées. Tel est le cas pour les taux de coissance selon les lequels pour la plupart des sites les plants non greffées inoculés donnent les meilleurs résultats en hauteur et en diamétre. L'inoculation n'a pas eu d'effet significatif. Les résultats de cette étude contribueront à une meilleure maîtrise des techniques de raccourcissement du cycle de production du baobab, par conséquent, au développement de politiques de rajeunissement des parcs à baobab au Sénégal pour mieux répondre à la demande du marché national et international de plus en plus croissant.

Mots Clés: Adansonia digitata L., Greffage, Inoculation, Baobab, Kolda, Sédhiou 


\title{
Effect of Horticultural Grafting and Mycorrhizal Inoculation on the Growth of Baobab (Adansonia Digital L.) in Upper and Middle Casamance, Senegal
}

\author{
Tamsir Mbaye, \\ (Chargé de Recherches, PhD, Phytogéographie) \\ Institut Sénégalais de Recherches Agricoles/Sénégal \\ Fatou Gning, \\ (Doctorant en Geoscience and Natural Resource Management) \\ Université de Copenhague \\ Dioumacor Fall, \\ (Chargé de Recherches, PhD, Microbiologie/physiologie des plantes) \\ Institut Sénégalais de Recherches Agricoles/Sénégal \\ Ababacar Ndiaye,
(Doctorant en Agroforesterie et Productions Végétales)
Université Assane Seck de Ziguinchor/Sénégal
Daouda Ngom,
(Professeur, PhD, écologie)
Université Cheikh Anta DIOP de Dakar/Sénégal \\ Mahani Cisse, \\ (Master, Agroforesterie), \\ World vision Sénégal \\ Saliou Ndiaye, \\ (Professeur, $\mathrm{PhD}$, Agronomie) \\ Université Thiès/ENSA/Sénégal
}

\begin{abstract}
The aim of this work is to study the effect of grafting and mycorrhizal inoculation on recovery, survival and success rates and on certain baobab growth variables in the Kolda and Sedhiou regions. To do this, a complete randomized experimental block consisting of four treatments on baobab plants (grafted inoculated, grafted uninoculated, ungrafted inoculated and ungrafted uninoculated grafted) was placed in eight (8) sites. Grafting was performed using the terminal slit method and inoculation with a mixture of archaeal mycorrhizal fungi strains (Glomus aggretatum, G. intraradices and G.
\end{abstract}


Fasciculatum) in the nursery. Two years after planting, the largest diameters and the largest number of branches are obtained with the grafted plants (inoculated and uninoculated) whereas the highest heights with those not inoculated (ungrafted and grafted). On the other hand, the number of leaves did not give any significant difference. Moreover, there are significant differences between the sites related inter alia to their mode of management. This is the case of the growth rates, which ungrafted inoculated plants have the more important diameter and height in most of the sites. There is no significant effect's inoculation. The results of this study will contribute to a better control of the techniques of shortening the cycle of production of baobab by horticultural grafting and, consequently, to the development of rejuvenation policies of baobab parks in Senegal to better meet the demand of the national market and more and more international.

Keywords: Adansonia digitata L., Grafting, Baobab, Biofertilization, Food security, Senegal

\section{Introduction}

Les arbres jouent un rôle important grâce aux multiples biens et services qu'ils rendent aux populations, surtout rurales. En effet, ils leurs fournissent des produits à usages multiples pour leur sécurité alimentaire et des revenus pour améliorer leurs conditions de vie.

Au Sénégal, les peuplements forestiers jouent un rôle alimentaire de premier ordre (Loubelot (2012), Sène (2001), Giffard(1974). En dehors des parcs à Faidherbia albida (Del) A. Chev. (Fabacées), Cordyla pinnata (Lepr. ex A.Rich.) Milne-Redh.(Fabacées),ceux de Adansonia digitata L. (baobab) sont les plus importants du Sénégal. En effet, le baobab est l'une des espèces caractéristiques du paysage agricole en zone sahélienne.

Pour son importance, l'homme l'a toujours conservé dans les champs de cultures (Cissé et Gning, 2013, Ndour et Gaye, 1995 ). Dans les régions de Kolda et de Sédhiou, toutes les parties du baobab sont utilisées quotidiennement par les communautés locales dans leur alimentation (Cissé et Gning, 2013).

Malgré cette importance, le vieillissement des parcs à baobab est prégnant (Ndiaye, 2013). Cette situation est liée, entre autres, à une faiblesse de la régénération naturelle, à la pression démographique, aux feux de brousse. En plus de cela, le baobab occupe une place marginale dans les campagnes de reboisement des pays du Sahel à cause de considérations culturelles (Cissé et Gning, 2013; Ces/Agf, 2000;Cisse, 1995).

Aussi, ces facteurs sont accentués par certaines pratiques agricoles, pastorales, médicinales (pharmacopée) et à l'artisanat qui mettent de plus en plus en danger la survie et la régénération des parcs à baobabs. En effet, la 
filière du baobab dans les régions de Kolda et de Sédhiou reste menacée à cause de l'émondage $(46 \%)$ qui affectent respectivement la production fruitière du baobab et l'écorçage $(76 \%)$ qui entrainela mortalité de plusieurs sujets (Cissé et Gning, 2013).

Ainsi, pour renouveler les parcs et répondre à la forte demande, la recherche forestière est interpellée. Dans ce cadre, un programme de recherche en milieu paysan a été déroulé de 2013 à 2016 dans les régions de Kolda et de Sédhiou au Sud du Sénégal. Il a consisté à l'utilisation du greffage et de l'inoculation mycorhizienne pour réduire substantiellement le cycle de production des fruits du baobab.

Contrairement aux espèces des grandes cultures dont la domestication et l'amélioration remontent à 3000 ans av. JC, celles forestières peuvent toujours être considérées comme sauvages malgré les améliorations depuis les années 1950. Ainsi, après la sélection suivant un certain nombre de critères, il reste à multiplier l'individu sélectionné à suffisance. Pour ce faire, seules les techniques de multiplication végétatives permettent une transmission fidèle des caractères désirés aux générations futures (Sbay H. et Lamhamedi M.S., 2015). Il s'agit de reproduire des copies semblables dont le génome est identique à la plante-mère. Toutefois, un tel processus nécessite l'utilisation de porte greffes jeunes et un personnel qualifié (Sbay H. et Lamhamedi M.S., 2015).

Par ailleurs, l'utilisation des phosphates naturels ou la mycorhization contrôlée peut améliorer la production des ligneux (Bajwa et al., 1988 ; Bâ et al., 2000). La mycorhization contrôlée est un ensemble de techniques qui consiste à isoler, cultiver, sélectionner, multiplier, incorporer et suivre le champignon dans le sol afin de produire des plants «biologiquement améliorés » par optimisation de la symbiose (Garbaye, 1991). Si ces techniques ont commencé à être transférées dans les régions tempérées ou dans certaines régions tropicales, elles ont fait l'objet de très peu d'études en Afrique de l'Ouest.

L'objectif de cet article est d'évaluer l'effet simultané du greffage et de l'inoculation mycorhizienne sur des paramètres de croissance $\left(\mathrm{HTP}^{1}, \mathrm{NR}^{2}\right.$, $\mathrm{DC}^{3}$ et $\mathrm{NF}^{4}$ ) du baobab en milieu paysan.

\footnotetext{
${ }^{1}$ Hauteur tige principale

${ }^{2}$ Nombre de rameaux

${ }^{3}$ Diamètre au collet

${ }^{4}$ Nombre de feuilles
} 


\section{Materiel et methodes}

\section{Présentation de la zone d'étude}

\section{Présentation de la zone d'étude}

L'étude a été réalisée dans sept sites dont deux de la commune de Saré Bidji (Dianabo et Boguel) dans la région de Kolda (Figure 1), un de la commune de Sakar (Bougnadou), trois de la commune de Bogal (Sénoba, Diop Counda et Fololo Birane) et un de la commune de Ndiamalathiel (Koboyel) dans la région de Sédhiou (Figure 1). Le climat de la zone d'étude est caractérisé par l'alternance d'une saison sèche de novembre à mai et d'une saison pluvieuse de mai à octobre. Les moyennes pluviométriques annuelles varient entre 800 et $1600 \mathrm{~mm}$ de 1985 à 2015 (Figure 2), avec de nombreuses amplitudes d'une année à l'autre. Les régions de Kolda et de Sédhiou disposent d'un énorme potentiel en termes d'espèces ligneuses et fourragères dont les essences varient en fonction de la zone. A Sédhiou, on rencontre la savane arborée, la palmeraie, la rôneraie ainsi que la mangrove localisée dans les bolongs et le long du cours d'eau Soungroungrou. A Kolda, en plus d'un important tapis de graminées, on trouve des espèces arbustives telles que Acacia macrostachya Reichenb. ex DC.(Fabacées), Oxytenantheraabyssinica(A. Rich.) Munro (poacées), etc. La présence de grands arbres sont notés dans cette zone notamment Adansonia digitata $\mathrm{L}$. (Baobab) de la famille des Bombacacées, selon la classification classique, ou des Malvacées, selon la classification phylogénétique, Daniellia oliveri (Rolfe) Huch. Et Dalz. (Césalpiniacées), Parkia biglobosa (Jacq.) R. Br. Ex G.Don (Mimosacées), Pterocarpus erinaceusPoir. (Fabacées), Cordyla pinnata (Lepr. Ex A. Rich.) Milne-Readhead (Fabacées), Sterculia setigera Del. (Sterculiacées selon la classification classique, ou des Malvacées selon la classification phylogénétique), etc. 

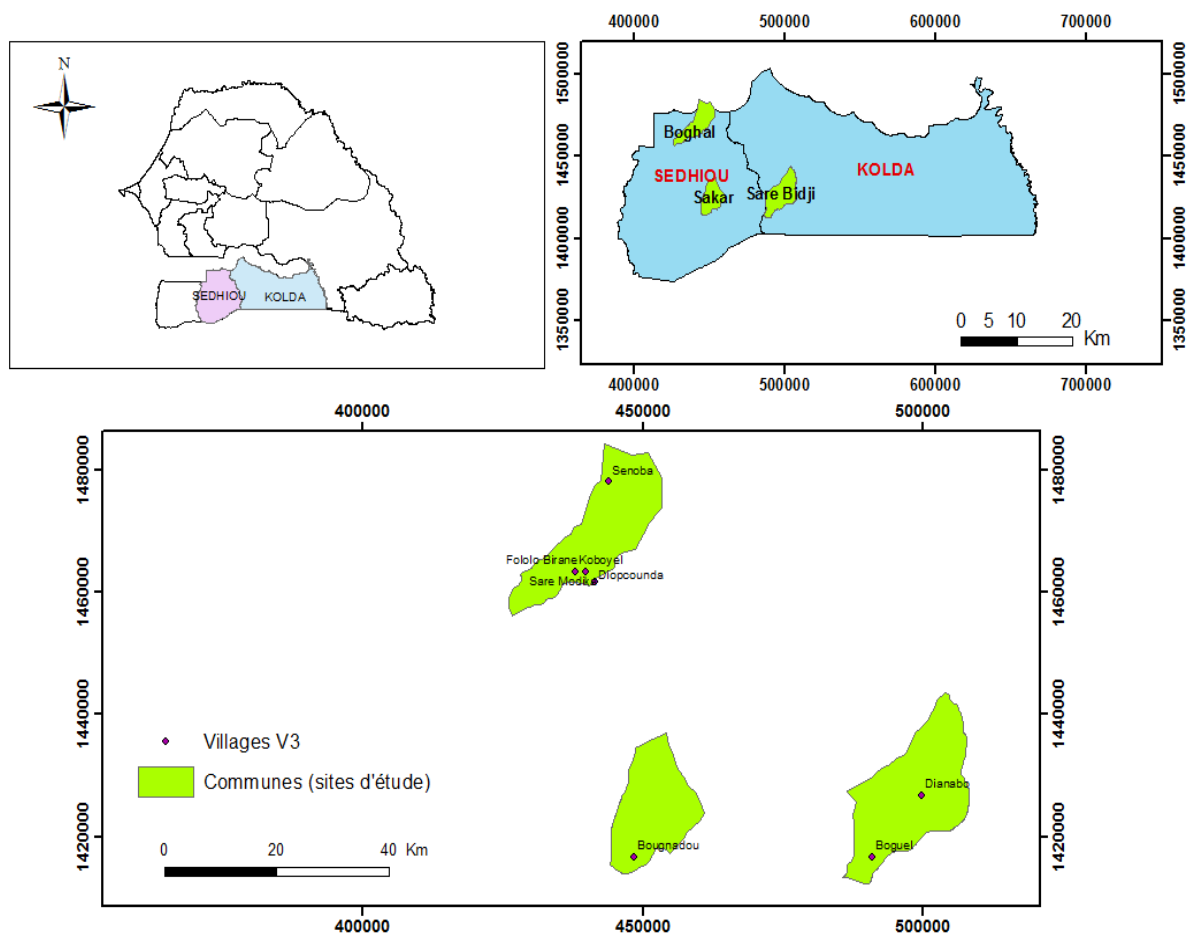

Figure 1 : Localisation de la zone d'étude

Cette zone est marquée par un relief de plateaux incisés par des vallées et les sols sont ferrugineux tropicaux, hydromorphes et limono-argilo-sableux.

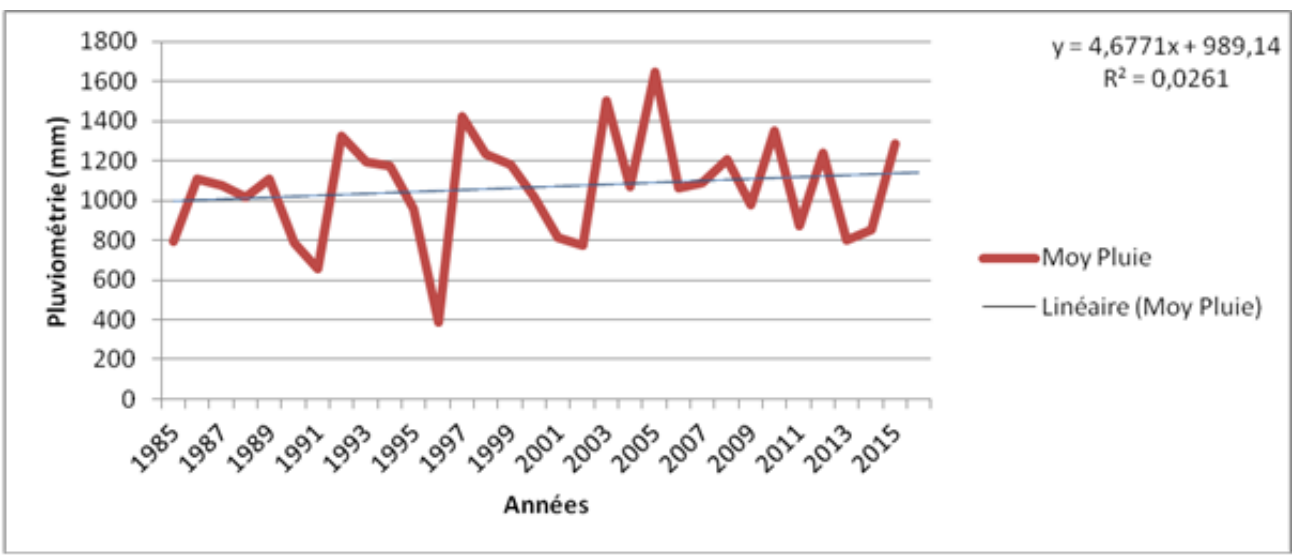

Figure 2: Pluviométrie de 1985-2015 : Kolda (Source de données : Agence Nationale de l'Aviation Civile et de la Météorologie, Sénégal)

\section{Production de porte-greffes}

Les porte-greffes ont été produits dans la pépinière du Centre National de Recherches Forestières (CNRF) de l'Institut Sénégalais de Recherches 
Agricoles (ISRA) à Dakar. D'abord, le rempotage de gaines avec un substrat de croissance constitué d'un mélange de terreau (2/3) et de sable (1/3) été effectué. Aussi, les graines, provenant des sept sites de transplantation dans les régions de Kolda et de Sédhiou, ont été prétraitées avec de l'acide sulfurique concentré $(96 \%)$ pendant 1 heure, puis trempées dans de l'eau froide durant 20 minutes. Ceci est dû pour lever leur inhibition tégumentaire, liée aux enveloppes séminales. Ensuite, après avoir mesuré l'inoculum, des poquets ont été creusés à l'intérieur de chaque gaine afin de mettre le mélange d'inoculum constitué de souches de champignons endomycorhiziens (Glomus aggretatum, G. intraradices et $G$. fasciculatum) à raison de $20 \mathrm{~g}$ par gaine contenant 100 spores. Ces souches ont été fournies par le Laboratoire Commun de Microbiologie IRD/ISRA/UCAD de Dakar-Sénégal (Certifié ISO 9001, version 2000). Enfin, après cette phase, le semis des graines a été effectué suivis de l'arrosage des gaines (qui sont de nature polyéthylène).

La fréquence d'arrosage a été tous les deux jours, pendant leur phase de développement jusqu'à la fin de l'expérimentation (9 mois).

\section{Greffage horticole}

Les greffons ont été prélevés sur des arbres « plus » qui ont été choisis par les populations des différents sites d'étude au mois de mai 2014. Ils ont été conservés dans des sacs en sisal mouillés pendant leur transport puis mis dans des réfrigérateurs pour au maximum 72 heures le temps de finir les opérations de greffage afin d'éviter leur déshydratation.

Le greffage a été réalisé 9 mois après semis du porte-greffe par la méthode de la fente terminale. Le porte-greffe a été coupé à $15 \mathrm{~cm}$ du collet, puis fendu diamétralement à l'aide d'un greffoir sur une profondeur de 3-4 $\mathrm{cm}$. Le greffon a subi une taille en biseau double à la base sur une hauteur de 3-4 cm (figure 3a) puis a ensuite été introduit dans la fente du porte-greffe (figure $3 b$ ). La jonction entre porte-greffe et greffon a été ligaturée à l'aide d'un ruban en cellophane (photo 3c). Après le greffage, les plants ont été mis dans des mini serres (figure $3 \mathrm{~d}$ ) durant 4 semaines afin de les protéger du vent et de leur permettre de conserver la chaleur pour faciliter la remontée de la sève.

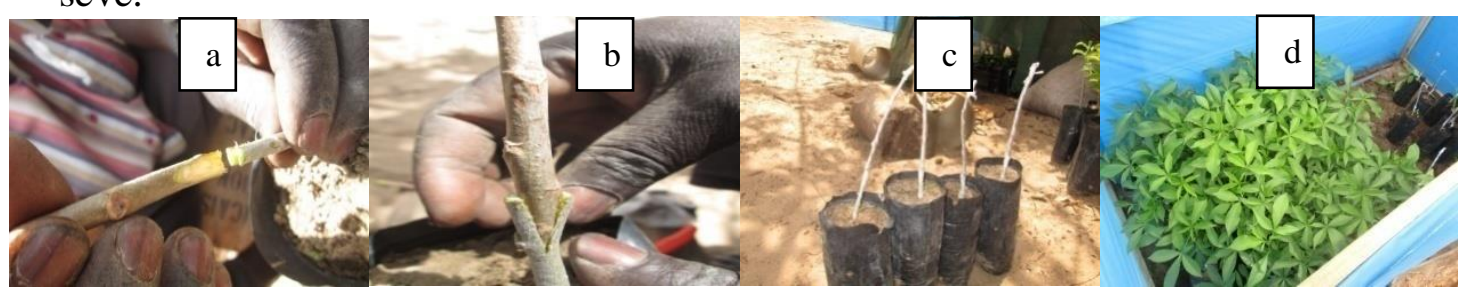

Figure 3 : Introduction du greffon dans le porte-greffe

$(a, b)$ ligature greffe (c) acclimatation (d) 


\section{Plantation}

Elle s'est déroulée dans les sept sites d'étude, pendant la saison des pluies (fin août 2014) sur des parcelles clôturées d'un demi- hectare, suivant un dispositif expérimental de Fisher ou bloc aléatoire complet de deux facteurs (greffage et inoculation), trois blocs (répétitions) et quatre traitements par bloc (greffé inoculé (GI), greffé non inoculé (GNI), non greffé inoculé (NGI), non greffé non inoculé(NGNI)) (figure 4). Ainsi, pour chaque bloc ou répétition, il y a 40 plants de baobab répartis comme suit : 20 plants non greffés (10 inoculés et 10 non inoculés) et 20 plants greffés (10 inoculés et 10 non inoculés). Au total pour les 3 répétitions, il y a 120 plants (avec 60 plants greffés et 60 plants non greffés) pour chaque site. L'écartement entre les lignes et entre les plants est de $7 \mathrm{~m}$ et une bordure d'un mètre a été laissée entre la clôture et les plants.

Bloc 1

Bloc2

Bolc3

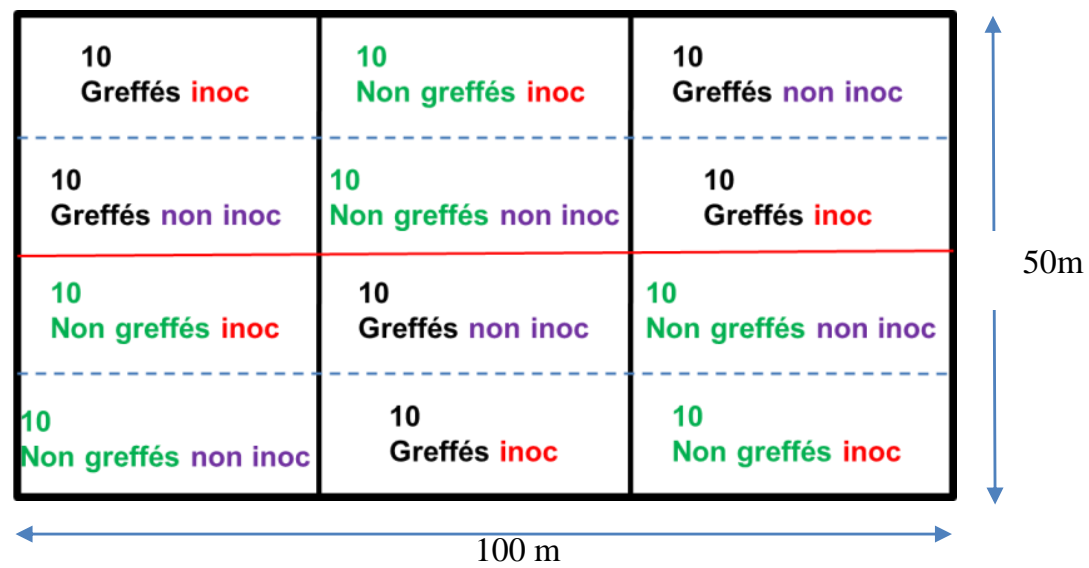

Figure 4 : Dispositif expérimental

La plantation a été effectuée (Figure 5b) après la délimitation des différents blocs de chaque site (Figure 5a).

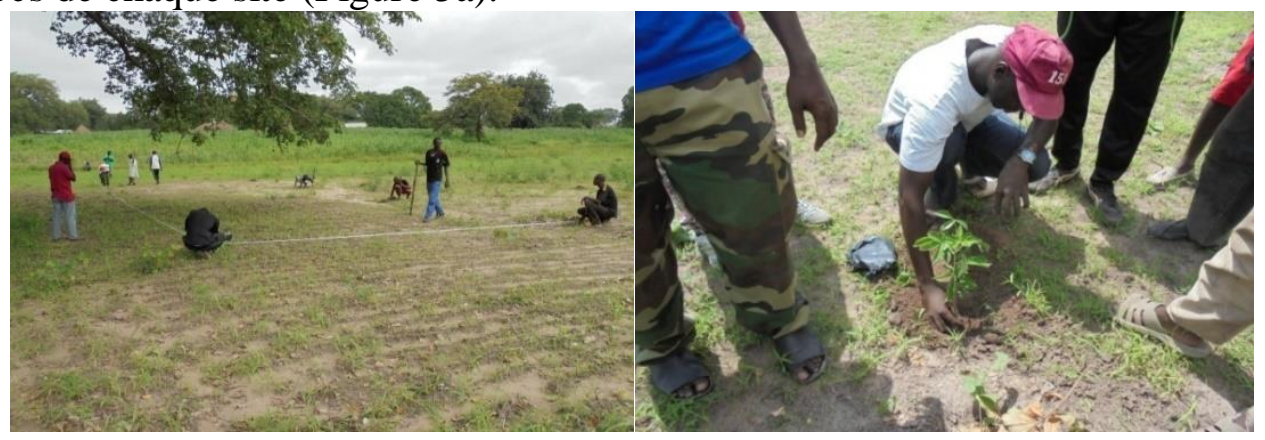

(a)

(b)

Figure 5 : Délimitation des blocs (a) et plantation (b) 
Après la plantation, la hauteur, le diamètre au collet, le nombre de rameaux et de feuilles ont été mesurés. Ces variables ont été suivies de nouveau après deux ans de plantation. La hauteur totale sur-pieds a été mesurée grâce à une règle graduée en mesurant de la base jusqu'au sommet du bourgeon terminal (Figure 6a). Le diamètre a été déterminé à la base, à l'aide d'un pied à coulisse (Figure 8c). Les nombres de feuilles et de rameaux ont été comptés manuellement (Figure 6b).

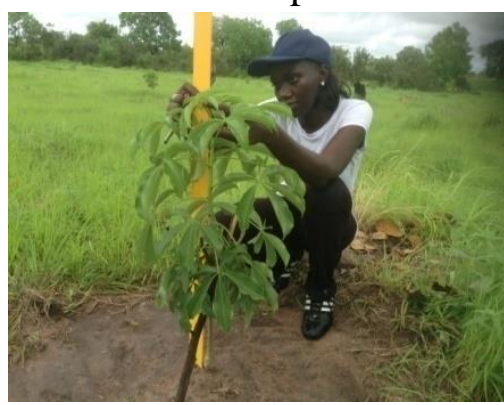

(a)

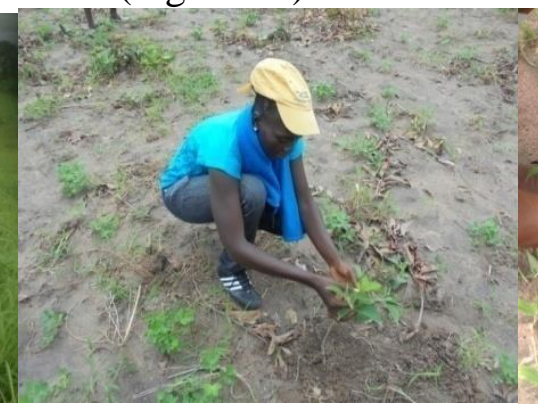

(b)

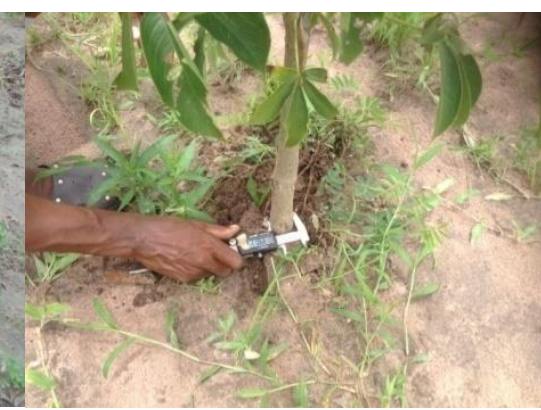

(c)

Figure 6: Mesure de la hauteur (a), décompte de feuilles (b) et mesure du diamètre (c)

\section{Traitements de données}

Les analyses ont été effectuées avec les logiciels Minitab 17 et R (version 3.2.0, 2015). Concernant les variables mesurées de croissance en l'occurrence la hauteur (HTP), le nombre de ramaux (NR), le diamétre au collet (DC) et le nombre de feuilles (NF), la normalite des données obtenues deux ans après plantation a été d'abord vérifiée. Le test paramétrique anova modèle linéaire a été appliqué sur les données normales après transformation (NR et DC) ou non (HTP). Par contre, les données et NF ne respectant pas la normalité même après transformation, le test non paramétrique anova médiane test a été utilisé. Les groupes homogènes ont été déterminés par le test de la plus petite différence de Fisher (LSD).

Egalement, pour chaque site, les taux de croissance en hauteur et en diamètre ont été calculés deux ans après plantation suivant la formule : $\mathrm{T}=[(\mathrm{St}$ - S0) /S0] x 100, où S0 est la taille initiale et St la taille finale. Une analyse de variance (modèle mixte: Bloc, facteur aléatoire et Greffage, Inoculation, facteurs fixes) a été effectuée sur les données. Le test de structuration de moyenne de Tukey a été utilisé pour séparer les moyennes dans le cas d'un effet significatif d'un facteur ou de l'interaction entre deux facteurs.

\section{Resultats}

Effet des traitements (greffage et inoculation avec ou sans) des plants sur les variables de croissance (HTP, DC, NF et NR).

Le Tableau 1 montre que les traitements et les sites ont chacun un effet significatif ( $\mathrm{p}$-value <0.05) sur toutes les variables de croissance. 
Tableau 1: résultats des différences de la HTP, DC et du NR par le model linéaire et celle du NF par le test de comparaison des médianes (médiane test), des plants de baobab par les sites et les traitements. P-value montre les différences significatives.

\begin{tabular}{|c|c|c|c|c|}
\hline Variables & HTP & NR & NF & DC \\
\hline P-value & p-value & p-value & p-value & p-value \\
\hline SITES & $0.0005997 * * *$ & $0.0037373 * *$ & $0.01726705 *$ & $0.002299 * *$ \\
\hline Traitements & $6.077 \mathrm{e}-07 * * *$ & $0.0001096 * * *$ & $0.01702267 *$ & $1.495 \mathrm{e}-06 * * *$ \\
\hline \multicolumn{4}{|c|}{$* * *$ différence très significative ; } \\
\hline \multirow{4}{*}{$*$ différence moyennement significative ; } \\
\hline
\end{tabular}

\section{Sur la hauteur}

L'analyse de la figure 7 ressort que la hauteur totale est plus élevée chez les plants non inoculés (non greffé (a) et greffé (b)) que chez les plants inoculés (c). L'inoculation n'influe pas positivement sur la croissance en hauteur aussi bien pour les plants greffés que pour ceux non greffés.

$\operatorname{HTP}(\mathrm{cm})$

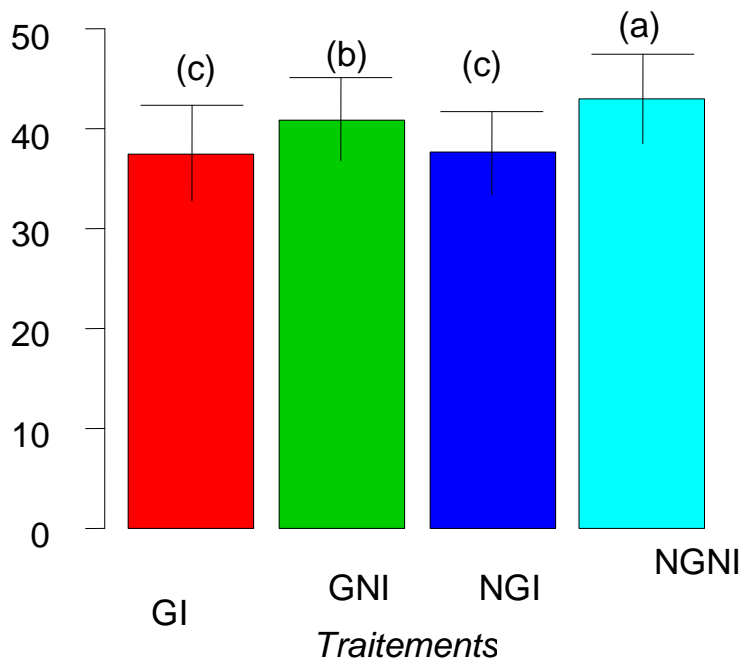

Figure 7: effet des traitements sur la croissance en hauteur

Les sites de Boguel, Dianabo et Sénoba présentent les hauteurs les plus élevées (a) suivis de

Folo Birane (ab), ensuite Bougnadou et Koboyel (bc) et enfin Diop Counda (c) (figure 8). 


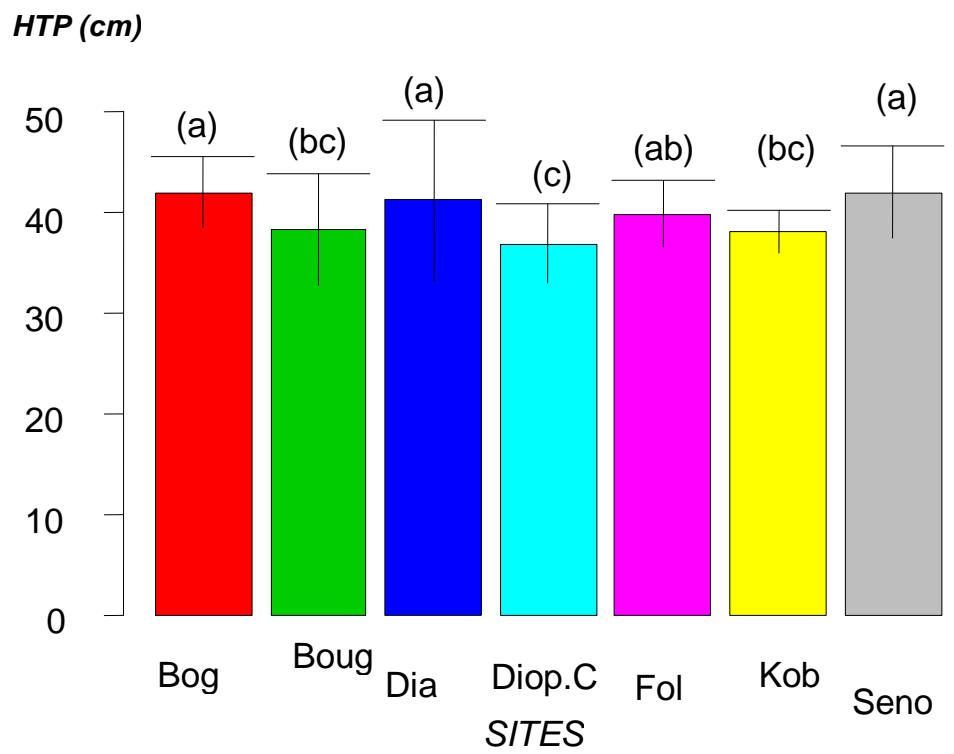

Figure 8: effet des sites sur la croissance en hauteur

\section{Sur le nombre de rameaux}

Selon la figure 9, les plants greffés(a) ont des nombres de rameaux plus importants que ceux non greffés (b).

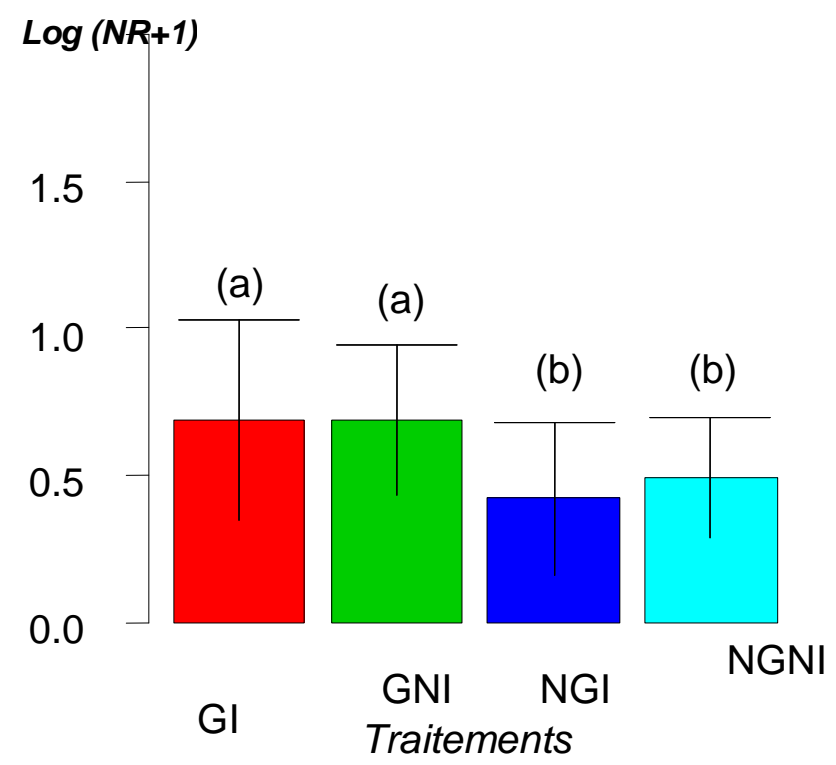

Figure 9 : effet des traitements sur le nombre de rameaux

La figure 10 indique que le plus grand nombre de rameaux est obtenu à Boguel (a) et Folo Birane (b), suivis de Dianabo (b) ensuite Bougnadou, Koboyel et Sénoba (bc) et enfin Diop Counda (c). 


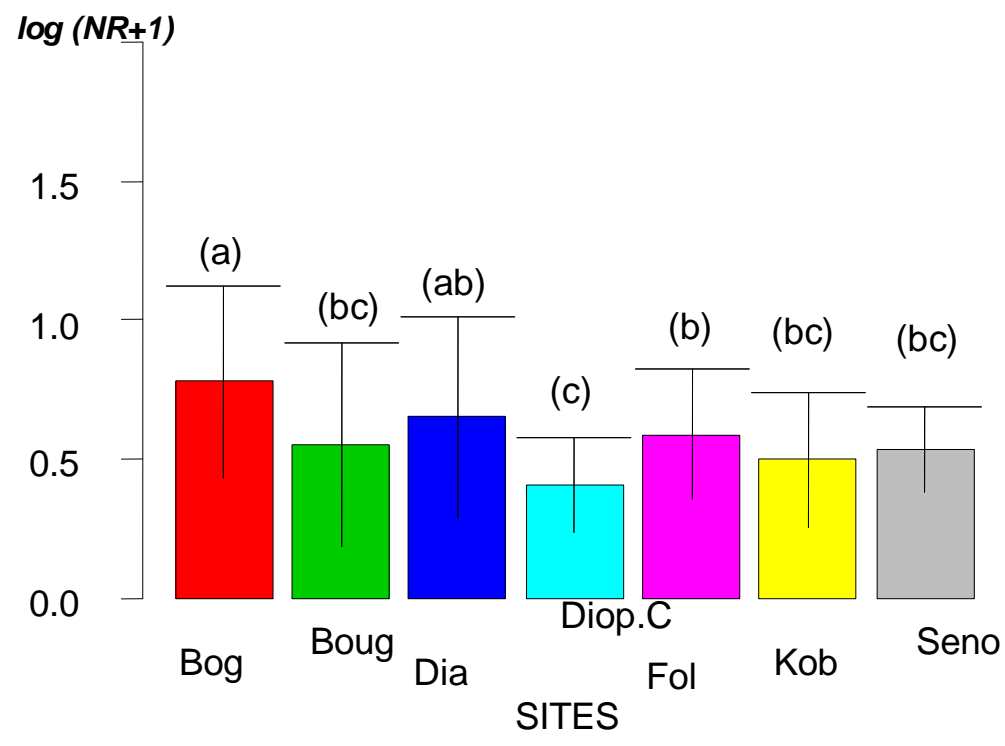

Figure 10 : effet des sites sur le nombre de rameaux

\section{Sur le diamètre au collet}

La figure 11 indique que les plants greffés ( $a$ et $a b$ ) présentent des diamétres au collet plus gros que ceux non greffés (bc et c).

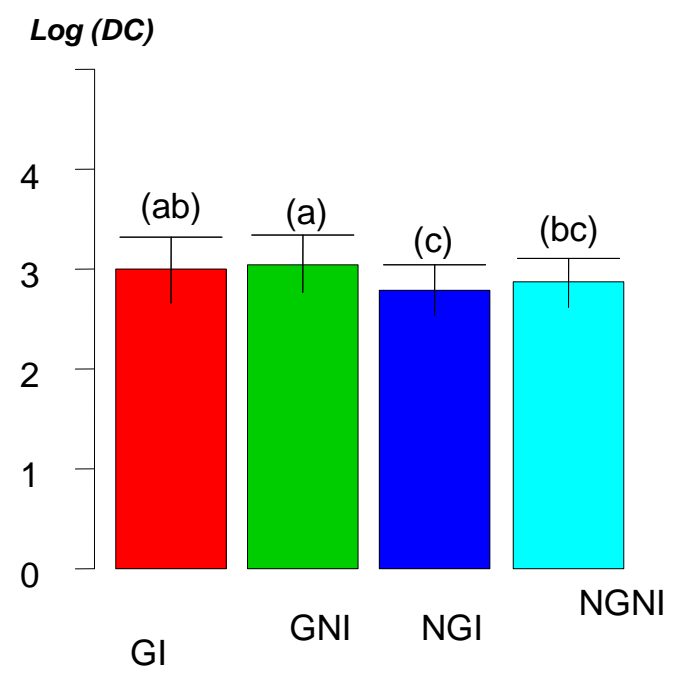

Traitements

Figure 11: Effet des traitements sur le diamètre au collet

Boguel et Folol Birane (a) ont les plus gros diamètre au collet, suivis de Diop Counda (ab) ensuite Sénoba (bc), Bougnadou, Koboyel (dc) et enfin Dianabo (d). 


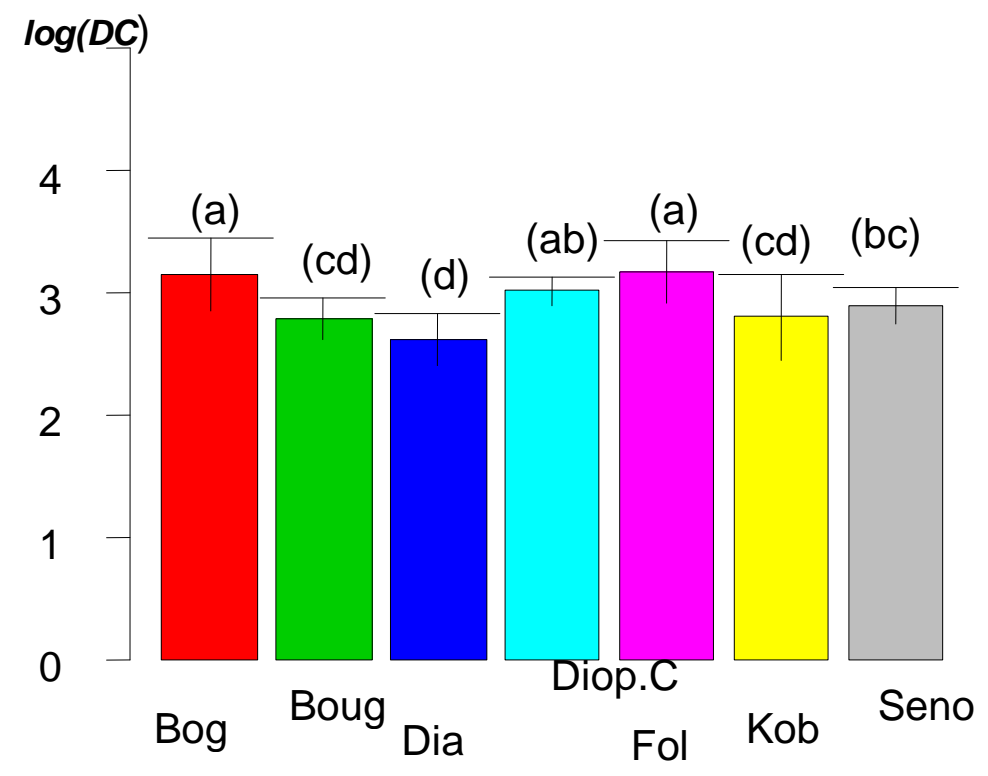

\section{SITES}

Figure 12: Effet des sites sur le diamétre au collet

\section{Sur le nombre de feuilles}

L'analyse de la figure 13 montre que les non greffés non inoculés (a) donnent le plus grand nombre de feuilles que les autres tritements (b).

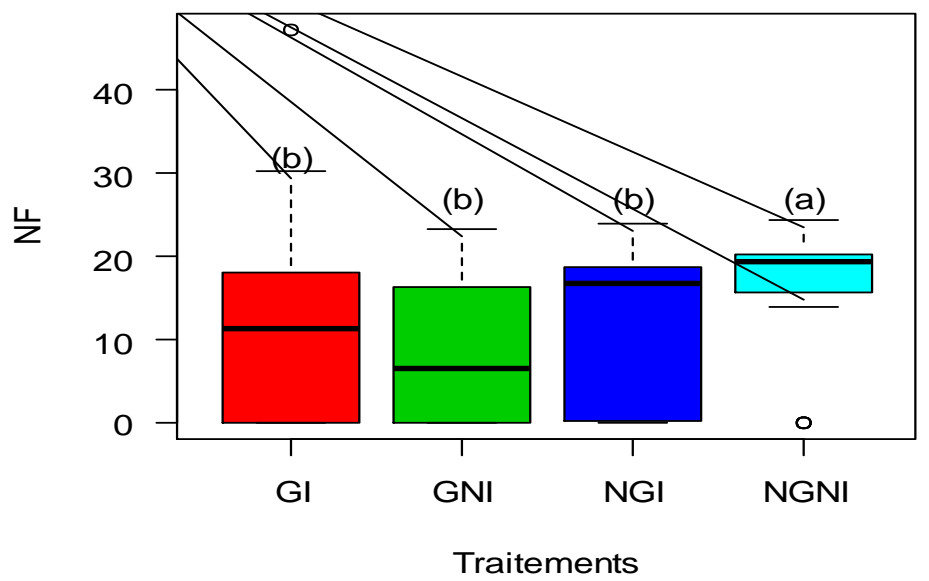

Figure 13: Effet des traitements sur le nombre de feuilles

Selon les sites, le plus grand nombre de feuilles est observé à Boguel (a), suivi de Bougndou et Sénoba (b),ensuite Folo Birane (bc) et enfin Dianabo, Diop Counda et Koboyel (c)

(Figure 14). 


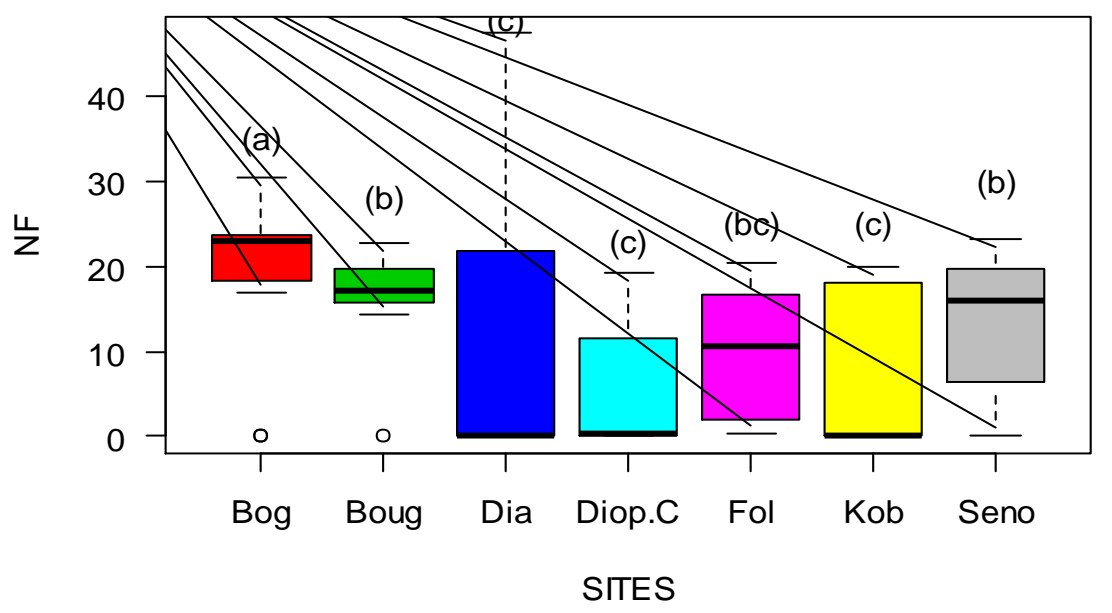

Figure 14: effet des sites sur le nombre de feuilles

Effet des traitements (greffage et inoculation avec ou sans) sur les taux de croissance en hauteur et en diamètre

\section{Sur la hauteur}

L'analyse de la figure 15 montre pour les traitements un effet significatif dans certains sites (Dianabo, Bougnadou, Sénoba et Diop Counda) et un effet non significatif dans d'autres sites (Boguel Fololo Birane et à Koboyel). Les taux de croissance les plus importants (>150\%) ont été obtenus à Boguel et Koboyel et les plus faibles $(<50 \%)$ à Dianabo pour tout traitement.

Dans les sites à différence significative entre les traitements, seul le traitement non greffé inoculé (NGI) se retrouve toujours dans le groupe «a », celui des valeurs les plus grandes du taux de croissance. 


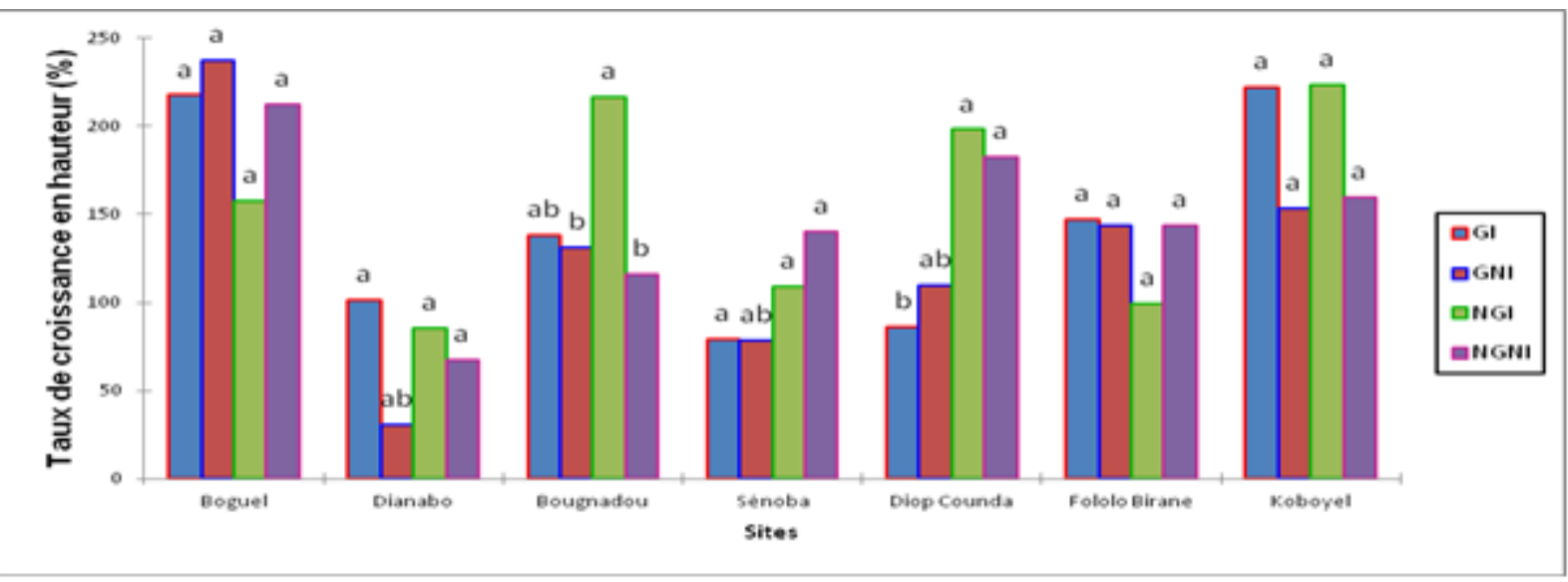

Pour chaque site, les valeurs moyennes suivies d'une même lettre ne sont pas significativement différentes au seuil de 5\% selon le test de Tukey

Figure 15 : Variation du taux de croissance en hauteur en fonction du greffage et de l'inoculation par site

\section{$\checkmark \quad$ Sur le diamètre}

La figure montre un effet significatif des traitements dans tous les sites sauf à Fololo Birane où il y a un seul groupe «a ». Les taux de croissance en diamètre sont élevés à Boguel, Bougnadou, Koboyel et à Fololo Birane (> 250\%), moyennement élevés à Diop Counda, à Sénoba et à Dianabo.

Dans tous les sites, le traitement non greffé inoculé (NGI) se retrouve le plus ( 5 sur 7 ) dans le groupe « a », celui des valeurs les plus grandes du taux de croissance en diamètre, que les autres traitements.

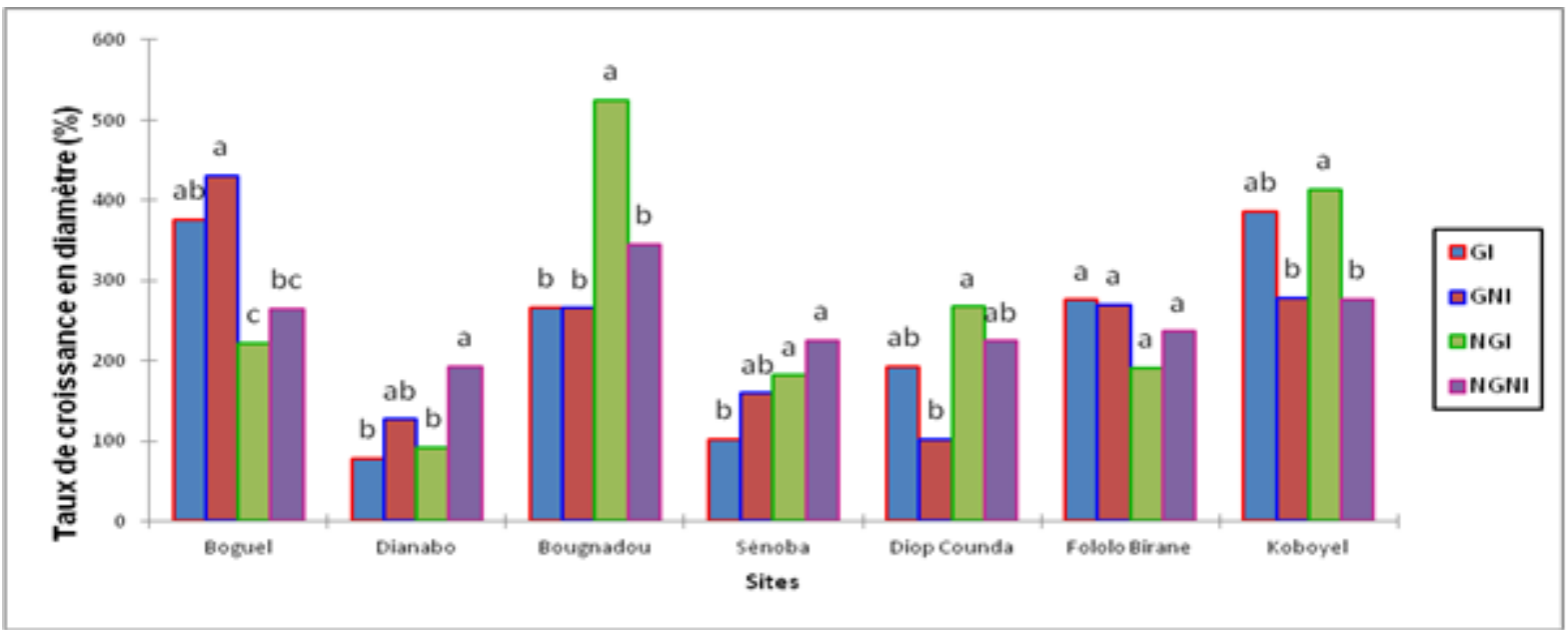

Pour chaque site, les valeurs moyennes suivies d'une même lettre ne sont pas significativement différentes au seuil de 5\% selon le test de Tukey

Figure 16: Variation du taux de croissance en diamètre en fonction du greffage et de l'inoculation par site 


\section{Discussion}

Pour ces plants jeunes de même âge obtenus par semis direct, deux ans après leur plantation, les mesures ont montré des différences significatives entre les traitements (greffage et inoculation ou sans) concernant les variables HTP, NR, DC et NF. Ainsi, nos résultats indiquent que les plants greffés présentent les diamètres les plus gros comparés aux plants non greffés. Cela pourrait s'expliquer par le fait que le greffage modifierait le fonctionnement physiologique des plants. En effet, en état jeune, l'absence des gourmands à la base entrainerait l'accumulation d'éléments nutritifs permettant une bonne croissance radiale de la plante (Ambang et al., 2009). Concernant la hauteur de la tige, nos résultats montrent que les plants non inoculés ont la hauteur la plus importante. Ce qui montre un effet négatif de l'inoculation sur la croissance en hauteur des plants. De même, Plenchette (1991) avait montré un effet dépressif sur la croissance de Acacia africana de l'inoculation. Et, son hypothèse explicative était qu'une partie des photosynthétats de la plante était alors détournée à son détriment par le champignon au contact des racines. Par ailleurs, entre les plants non inoculés ceux non greffés sont plus hauts que ceux greffés. Cela pourrait s'expliquer par le fait que ces plants non greffés soient issus de semis direct et n'ont pas pu développer beaucoup de ramifications après plantation. Aussi, ces plants n'avaient pas subi de coupe contrairement aux plants greffés. Ces résultats confirment les travaux de Bidima (2006) sur le manguier qui montre que le greffage permet d'avoir des arbres courts et accessibles. En effet, les arbres issus de semis direct croissent en hauteur, contrairement aux arbres greffés qui présentent un port ramassé intéressant pour les commodités de récolte. Ce qui justifie d'ailleurs les résultats montrant le plus grand nombre de rameaux chez les plants greffés contrairement aux non greffés.

Les taux de croissance calculés à partir de la plantation jusqu'à deux ans ont montré que les plants non greffés inoculés se retrouvent dans le groupe (a) des pants qui croissent plus rapidement en diamètre et en hauteur que les autres plants dans la plupart des sites. Cela prouve de l'effet positif de l'inoculation sur la croissance des pants non greffés. Bâ et al., (1996) ont montré que l'inoculation n'était bénéfique que si les souches utilisées sont plus compétitives que celles existantes dans le sol. Par ailleurs, l'effet négatif de l'inoculation trouvé ci-haut sur la croissance en hauteur pourrait être dû pendant la phase de pépinière. Aussi, deux ans après plantation, les plants inoculés n'ont pas pu rattraper en hauteur ceux non inoculés malgré leur plus important un taux de croissance après la plantation.

Par ailleurs, dans le cas où l'effet de l'inoculation soit négatif, il pourrait s'expliquer par les caractéristiques physicochimiques du sol. En effet, le sol pourrait contenir un taux élevé en éléments nutritifs comme le phosphore et l'azote qui inhibent la symbiose. Cet effet peut être dû aussi à une faible 
dépendance mycorhizienne de la plante. L'effet négatif de l'inoculation endomycorhizienne dans certains sites peut être dû aussi à une prédominance de la mycorhization naturelle.

Les résultats obtenus mettent en évidence également une différence de croissance des plants entre les sites d'étude comme l'indique le tableau 1. Cette différence est plus remarquable à Boguel qui a les hauteurs, les diamètres, les nombres de rameaux et de feuilles plus importants (groupe a). Cet effet, s'expliquerait par le mode de gestion appliqué aux plants dans les différents sites. En effet, dans les sites comme Boguel, où des cultures (arachide, niébé, piment et melon) ont été associées aux plants de baobab, un bon développement des plants a été noté. Ce constat est en phase avec les activités de gestion sylvicole notées au Mali et qui ont donné des résultats probants (CNRF/ISRA, 2013). Par contre, le site de Diop Counda a les plus faibles hauteurs, nombres de rameaux et de feuilles et celui de Bougnadou les plus faibles diamètres. Egalement, les plus faibles taux de croissance en hauteur et en diamètre sont observés à Dianabo. Dans ces sites, les parcelles n'ont pas été cultivées, ni désherbées. Par ailleurs, les faibles diamètres trouvés à Bougnadou pourraient être dû au manque de gestion des gourmands comme l'ont montré les travaux de Niang et al., (2010) sur l'influence négative des gourmands sur le greffage.

\section{Conclusion}

Ce travail a permis d'évaluer l'effet du greffage et de l'inoculation sur la croissance des plants de baobab en milieu paysan. L'étude a montré que les gros diamètres et le plus grand nombre de rameaux sont observés sur des plants greffés. Le plus grand nombre de feuilles est obtenu avec les plants non inoculés et non greffés. Pour ce qui est de la hauteur, elle est plus favorable aux plants non inoculés. Par contre, l'inoculation mycorhizienne n'a pas eu un effet positif sur la plupart des variables étudiées dans les différents sites. De même, une différence de comportement des plants entre les différents sites d'étude, liée à leur gestion, a été notée suivant les formes d'organisation des communautés (individuelle ou collective).

Ces résultats sont très importants dans une perspective de rajeunissement des parcs à baobab au Sénégal devenus trop vieux et faiblement productifs malgré une demande du marché de plus en plus croissante.

\section{Remerciements}

Nous remercions le Fonds national de Recherches agricoles et agroalimentaires (FNRAA) et le Programme de Productivité agricole en Afrique de l'Ouest (PPAAO) pour avoir financé les travaux. 


\section{References:}

1. Ambang Z., Amougou A., Ndongo B., Nantia J., Chewachong g.m., 2009. Résistance à la mosaïque virale de Manihot glaziovii par greffage sur M. esculenta TROPICULTURA, 27, 1, 8-14 pp.

2. Bâ A.M., Plenchette C., Danthu P., Duponnois R., Guissou T. Functional compatibility of arbuscular mycorrhizae with thirteen tropical fruit trees in Senegal, Agroforest. Syst. 95 (2000) 95-105.

3. Bâ A. M., Dalphe Y. \& Guissou T. 1996. Les Glomales d'Acacia holosericea et d'Acacia mangium. Bois et Forêts des Tropiques, 250 : 5-18.

4. Bajwa G.S., Sandhu H.S., Bal J.S. Effect of pruning severety on growth and bearing of ber, Indian J. Hortic. 22 (1988) 203-206.

5. Bationo B.A., Compaore et Niang A., 2003. Les parcs à baobabs dans le Plateau Central du Burkina Faso : structure et contraintes socioculturelles à la régénération in Leçons tirées des expériences de lutte contre la désertification au Sahel : Actes des travaux de l'Atelier sous - régional d'échange et de réflexion organisé par le Centre de recherches pour le développement international (CRDI), 12-16 juillet 2004, Saly Portudal, Sénégal ; 72-79pp.

6. Bationo B.A., Lamien N., Demers N., Kandji S., 2009. Culture du baobab Adansonia digitata L. (Bombacaceae) en planche maraîchère : une méthode pour simplifier sa récolte et favoriser sa propagation au Sahel. 1-8pp.

7. Bidima I. M., 2006. Le greffage des arbres fruitiers: Agrumes, Manguiers, Avocatiers, Fiche technique 1-13pp

8. Bâ A. M., Guissou T., Duponnois R., Plenchette C, Sacko O., Sidibé, 2002. Mycorhization contrôlée et fertilisation phosphatée : applications à la domestication du jujubier. J. Fruits.

9. CES/AGF, 2000. Evaluation du taux de survie des plantations dans le plateau central du Burkina Faso, UCP/CES/AGF, Yako, 38 p.

10. Cissé M. et Gning F., 2013. Les parcs agroforestiers à Adansonia digitata L. (Baobab) en Haute et Moyenne Casamance : opportunités et contraintes. Mémoire de licence, Université Assane SECK de Ziguinchor, 40pp.

11. Cissé M. I., 1995. - Les parcs agroforestiers au Mali: état des connaissances et perspectives pour leur amélioration. ICRAF/AFRENA, Bamako, $53 \mathrm{p}$

12. Cissé M., 2015. Analyse des perceptions locales et des déterminants socio-économiques de l'adoption de technologies agroforestieres : Cas des planches maraichères et du greffage horticole d'Adansonia digitata L. (Baobab), Mémoire Master Université Assane SECK de Ziguinchor, 41pp. 
13. CNRF/ISRA, 2013. Rapport annuel projet FNRAA/BAOBAB, Institut sénégalais de Recherches agricoles (ISRA), Centre national de recherches forestières (CNRF), 20 pp, Sénégal.

14. Collière P., 2002. La forêt de Baobab (Adansonia digitata L.) de Nguekhokh (Sénégal) : analyse des causes de la dégradation de la forêt et recherche de solutions In chaîne et Baobab, Rapport d'étude de la mission humanitaire réalisée au CIAF de Nguekhokh du 27 juin au 21 août 2002 par Pierre Collière, 34pp

15. Danthu P., Soloviev P., 2000. Propagation par greffage de trois espèces forestières fruitières des zones tropicales sèches : Adansonia digitata, Balanites aegyptiaca et Tamarindus indica, Le Flamboyant 53 (2000) 22-24pp.

16. D., Sylla K. et Windou B., 2012. Mycorhization contrôlée et fertilisation phosphatée Applications à la domestication du jujubier, arbre fruitier forestier sahélien, In La grande muraille verte: capitalisation des recherches et valorisation des savoirs locaux, p. 251262

17. Djaha J.B.A., N'da adopo, A. A., Koffi E.K., Koffi C., Ballo C.K. et Coulibaly M., 2012. Croissance et aptitude au greffage de deux génotypes d'anacardier (Anacardium occidentale L.) élites utilisés comme porte-greffe en Côte d'Ivoire, Int. J. Biol. Chem. Sci. 6(4): 1453-1466, August 2012.

18. Garbaye J., 1991. Utilisation des mycorhizes en sylviculture, in : Lavoisier (Éd.), Les mycorhizes des arbres et plantes cultivées, Paris, France, pp. 197-248.

19. Giffard P. L., 1974. L'arbre dans le paysage sénégalais-sylviculture en zone tropicale sèche, Tom 1. Centre technique forestier tropical, Sénégal, Dakar, 271pp.

20. LOUBELOE., 2012.Impact des produits forestiers non ligneux (pfnl) sur l'économie des ménages et la sécurité alimentaire : cas de la république du Congo.

21. Ndiaye A., 2013. Caractérisation biophysique des parcs à Adansonia digitata L. (Baobab) en Moyenne et Haute Casamance : dynamique, production fruitière et anthropisation, département agroforesterie, université Assane Seck de Ziguinchor Sénégal, 58 p.

22. Niang D., Gassama Y. K., Ndiaye A., Sagna M. Ndiaye S. A. S., et Toure M. A., 2010.In vitro micrografting of Sterculiasetigera Del. African Journal of Biotechnology Vol. 9(50), pp. 8613-8618, 13 December, 2010, Available online at http://www.academicjournals.org/AJB $\quad$ ISSN $1684-5315$ (C2010 Academic Journals. 
23. Plenchette C., 1991. Utilisation des mycorhizes en agriculture et horticulture, in : Lavoisier (Éd.), Les mycorhizes des arbres et plantes cultivées, Paris, France, 131-179.

24. Plenchette C., Fortin J.A., Furlan V., 1983. Growth responses of several plant species to mycorrhizae in a soil of moderate P-fertility, I : mycorrhizal dependency under field conditions, Plant Soil 70 (1983)

199-209.

DOI : 10.1007/BF02374781PRDI., 2013. Plan Régional de Développement intégré de Sédhiou de «2013-2018 »108p.

25. Samba N. A. S., Gaye A., Fall S.T., Diouf M., Diallo I., 2003. Le baobab : nouvelle plante maraichère du sahel. Fiche technique, ISRA, CORAF /WECARD, FNRAA, 6p.

26. Sbay H. Et Lamhamedi M.S., 2015. Guide pratique de multiplication végétative des espèces forestières et agroforestières: techniques de valorisation et de conservation des espèces à usages multiples face aux changements climatiques en Afrique du Nord.

27. Sène A., 2001. Rapport sur l'Exploitation et Valorisation des produits forestiers non ligneux dans la région de Kolda : Caractérisation des acteurs de base. Institut Sénégalais de Recherches Agricoles (ISRA), en collaboration avec le Bureau d'Analyses Macro-économiques (BAME), sous la coordination de l'UICN. 30 p.

28. Smith S.E., Read D.J., 1997. Mycorrhizal symbiosis, Academic Press, second edition, Oxford, UK.

29. Toure M.A., 2009., Caractérisation de la gommose et conservation des ressources génétiques de Sterculia Setigera Del. Thèse de Doctorat de 3éme cycle de biologie végétale ,113pp.

30. Toure, M.A., Diatta M., Mallou G., 2015. Anacardium occidentale L. multiplication par greffage horticole, document interne ISRA, 3p. 